\title{
METFORMIN - A CONVENIENT ALTERNATIVE TO INSULIN FOR INDIAN WOMEN WITH DIABETES IN PREGNANCY
}

\author{
LAVANYA RAI, MEENAKSHI D, ASHA KAMATH ${ }^{1}$
}

\section{ABSTRACT}

OBJECTIVE: To compare the use of metformin with that of insulin for the treatment of gestational diabetes mellitus (GDM) and type 2 diabetes mellitus (T2DM) unresponsive to diet therapy. MATERIALS AND METHODS: In this prospective observational study, maternal glycemic control and perinatal outcome in diabetic pregnancies were compared between 2 obstetric units, one using insulin therapy and the other using metformin therapy. Baseline pretreatment glycemic profile was done and then repeated weekly throughout pregnancy. The outcome measures studied were glycemic control, maternal complications and perinatal outcome. RESULTS: Sixty women with gestational and type 2 diabetes were enrolled, 30 each for metformin and insulin. Both groups were comparable with respect to age, body mass index (BMI), parity and pretreatment plasma glucose levels. Glycemic control was better with metformin after 1 week of therapy and also throughout gestation $(P=0.03-0.007)$. There were no major complications or perinatal deaths in this study. Mean gestational age and birth weight $(2.9 \pm 0.4 \mathrm{~kg}$ versus $3.1 \pm 0.4 \mathrm{~kg}, P=0.30)$ were comparable. However, there was a significant increase in neonatal intensive care unit (NICU) admission and stay for babies born in the insulin group. The cost of treatment was tenfold higher in thethe insulin group. CONCLUSION: Metformin is clinically effective, cheap and a safe alternative to insulin therapy in pregnant diabetic women.

Key words: Gestational diabetes, insulin, insulin resistance, metformin, type 2 diabetes in pregnancy

DOI: $10.4103 / 0019-5359.58878$

\section{INTRODUCTION}

Diabetes complicating pregnancy is on the rise, especially in south Asian countries like

\footnotetext{
Departments of Obstetrics and Gynecology, Manipal, Karnataka, India

Correspondence:

Dr. Lavanya Rai

Department of Obstetrics and Gynecology,

Kasturba Medical College Hospital,

Manipal, Karnataka, India

E-mail: railavanya@yahoo.com
}

${ }^{1}$ Biostatistics, Kasturba Medical College Hospital,
India. This is due to increasing incidence of gestational diabetes mellitus [GDM] and lower age at onset of type 2 diabetes mellitus [T2DM] in India ${ }^{[1]}$ Diet and insulin have been the main therapeutic agents for diabetic pregnancies for years. Oral hypoglycemic agents like glyburide and metformin have now been found to be useful and safe in pregnancy. Insulin therapy in pregnancy was considered best because of its efficacy, inability to cross the placenta and fetal safety. However, frequent injections, 
risk of hypoglycemia and cost are some of the disadvantages that stimulated interest in trials of oral hypoglycemic drugs in pregnancy.

\section{Objective}

To compare the use of metformin with that of insulin in the management of diabetic pregnancies.

\section{MATERIAL AND METHODS}

\section{Study design}

In this prospective observational study, one obstetric unit used metformin for GDM and T2DM in pregnancy. The results were compared with patients of another unit using only insulin. Approval for the study was obtained from the ethical committee after obtaining informed consent from participants.

Patients diagnosed with GDM after $100 \mathrm{~g}$ oral glucose tolerance test (GTT) following a positive glucose challenge test ( $\geq 140 \mathrm{mg} /$ $\mathrm{dL}$ ) with $50 \mathrm{~g}$ of oral glucose were included. GDM was diagnosed with 2 or more abnormal values in GTT as per Carpenter and Coustan's criterion $^{[2]}$ (fasting plasma glucose $\geq 95 \mathrm{mg} / \mathrm{dL}$, at 1 hour $\geq 180 \mathrm{mg} / \mathrm{dL}$, at 2 hours $\geq 155 \mathrm{mg} / \mathrm{dl}$, at 3 hours $\geq 140 \mathrm{mg} / \mathrm{dL}$ ). Women with T2DM in pregnancy and those with GDM who failed diet therapy of 1 week were recruited to receive either metformin or insulin therapy depending on the obstetric unit they belonged to. Diet therapy was advised by the same nutritionist for both the groups. Exclusion criteria were patients with deranged liver or kidney functions and those with type 1 diabetes. Metformin was given at a dose of $500 \mathrm{mg}$ three times a day to a maximum of $2000 \mathrm{mg} /$ day based on glycemic profile.
Insulin was similarly started and titrated based on plasma glucose values. Intermediateacting insulin (Mixtard) was the insulin used, and short-acting insulin (Actrapid) was used whenever required.

Glycemic profile ffasting (FG), 2 hours postbreakfast (PB), 2 hours post-lunch (PL) and 2 hours post-dinner (PD) glucose was done prior to therapy and weekly thereafter to monitor the effect of therapy. The goal of therapy was to have $F G$ around $<100 \mathrm{mg} /$ $\mathrm{dL}$ and 2 hours postprandial of $<130 \mathrm{mg} / \mathrm{dL}$. Plasma glucose was estimated by glucose oxidase method.

Maternal glycemic control constituted the primary outcome measure, while perinatal outcome was the secondary outcome measure for this study.

Patients were also monitored in the antenatal period for diabetes-related complications such as preeclampsia, urinary tract infection (UTI), vaginitis, fetal growth disorders, polyhydramnios, preterm labor and hypoglycemia (glucose $<60 \mathrm{mg} / \mathrm{dL}$ ). Macrosomia was defined as birth weight $\geq 4000$ g. 'Large for gestational age' (LGA) was weight $>90^{\text {th }}$ percentile, while weight $<10^{\text {th }}$ percentile was considered as 'small for gestational age' (SGA). All babies were monitored for hypoglycemia (glucose $<40 \mathrm{mg}$ / $\mathrm{dL}$ ) for 24 hours. NICU admission was done for neonatal morbidity such as birth asphyxia, birth injuries, hypoglycemia, hyperbilirubinemia (serum bilirubin $>12 \mathrm{mg} / \mathrm{dL}$ ), polycythemia (hematocrit $>60 \%$ ) and hypocalcemia (serum calcium $<8.0 \mathrm{mg} / \mathrm{dL}$ ). 
Table 1: Comparison of demographics of metformin and insulin groups

\begin{tabular}{lccc}
\hline Group & Metformin $(n=30)$ & Insulin $(n=30)$ & $P$ value \\
\hline Age $(\mathrm{y})$ & $30.7 \pm 3.8$ & $30.5 \pm 3.7$ & $0.77^{\star}$ \\
BMI $\left(\mathrm{kg} / \mathrm{m}^{2}\right)$ & $25.8 \pm 3.5$ & $26.4 \pm 4.9$ & $0.63^{\star}$ \\
Parity Primi & $14(47 \%)$ & $14(47 \%)$ & \\
Multi & $16(53 \%)$ & $16(53 \%)$ & $1^{\dagger}$ \\
Singleton gestation & $29(96.6 \%)$ & 30 & 0 \\
Twin gestation & $1(4.4 \%)$ & $6(20 \%)$ & $0.254^{\dagger}$ \\
Type 2 DM & $2(6.7 \%)$ & $24(80 \%)$ & $1^{\dagger}$ \\
Gestational diabetes & $28(93.3 \%)$ & $3(18.7 \%)$ & $0.299^{\ddagger}$ \\
GDM in previous pregnancy & $2(12.5 \%)$ & $19(63.3 \%)$ & $1^{\dagger}$ \\
POG at diagnosis of GDM & $14(47 \%)$ & $5(16.6 \%)$ & $0.146^{*}$ \\
( $\geq 28$ weeks) & $4(13.3 \%)$ & & $0.707^{*}$ \\
Bad obstetric history & & $114.5 \pm 21.3$ & \\
GTT Values (mg/dL) & $107 \pm 12.5$ & $194.5 \pm 35.4$ & \\
Mean Fasting & $191 \pm 27$ & & \\
Mean Post glucose load 1,2,3 h & & & \\
\hline
\end{tabular}

POG: period of gestation

$\mathrm{P}$ value $<0.05$ considered significant; ${ }^{*}$ mean $\pm 2 S D$, independent $t$ test; ${ }^{\dagger}$ Fisher's exact test; ${ }^{\circledR}$ Pearson's $X^{2}$

\section{Statistical analysis}

SPSS version 15 was used. For analysis, ANOVA test, Chi-square test, Fisher's exact test (when cell size is less than 5 ) and analysis of covariance were applied wherever appropriate. Statistical significance was accepted at $P<0.05$.

\section{RESULTS}

In this prospective study conducted over a period of 2 years (September 2004 to August 2006), 60 women with GDM and T2DM fulfilled the inclusion criteria. Table 1 shows the comparison between 2 groups of diabetic mothers treated with metformin and insulin.

Both groups were comparable with respect to age, BMI, parity and the GTT values. T2DM women in both groups (2 in metformin group and 6 in insulin group) continued their preconception therapy through pregnancy. Bad obstetric history for this study was defined as history of previous two abortions or one fetal

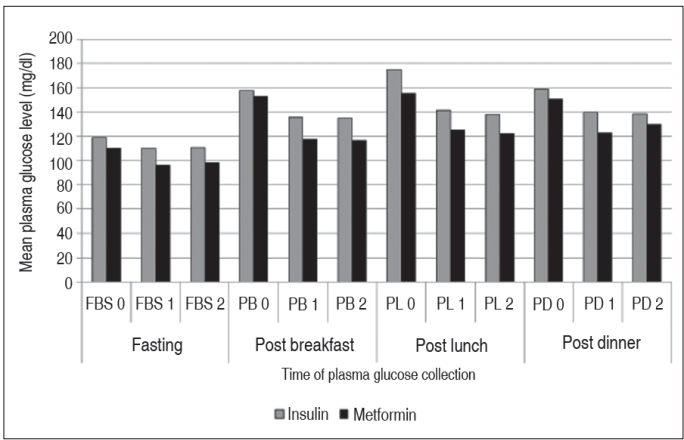

Figure 1: Pretreatment glycemic profile, adjusted mean glucose values after 1 week of therapy and all through pregnancy, FBS: fasting blood sugar; 0: pretreatment glucose value; PB: post-breakfast 1: adjusted mean glucose values after 1 week of therapy; PL: post-lunch; 2: adjusted mean glucose values throughout pregnancy; PD: post-dinner

\section{Actual values of Figure 1}

\begin{tabular}{lcc}
\hline & Insulin & Metformin \\
\hline FBS0 & $119.1+3.9$ & $110.1+2.6$ \\
FBS1 & $110.1+3.8$ & $96.1+1.7$ \\
FBS2 & $111+3.9$ & $98.3+1.8$ \\
PB0 & $157.4+6.9$ & $152.9+7$ \\
PB1 & $135.5+3.1$ & $117.6+3.3$ \\
PB2 & $135.3+3.5$ & $116.7+2.5$ \\
PL0 & $175.3+10$ & $155.1+6.3$ \\
PL1 & $141.6+3.7$ & $125+4.5$ \\
PL2 & $137.9+4$ & $122.1+2.4$ \\
PD0 & $158.9+7.2$ & $150.6+4.5$ \\
PD1 & $140+4.6$ & $122.7+2.9$ \\
PD2 & $138.6+3.8$ & $130.1+3.5$ \\
\hline
\end{tabular}


death or neonatal death due to obstetric reasons.

Glycemic profiles - before therapy, after 1 week of therapy and throughout pregnancy are depicted in Figure 1. Though the values in the insulin group before therapy appear marginally higher, the difference was not statistically significant $(P=0.08-0.69 ; 95 \% \mathrm{Cl}$, 1.04-26.2). To avoid its influence on immediate sugar control, adjusted mean values (analysis of covariance) were used in both groups while comparing the glucose values after starting therapy. A significant reduction in adjusted mean glucose levels in the metformin group was noted as compared to the insulin group $(P=<0.001-0.003 ; 95 \% \mathrm{Cl}, 5.2-29.7)$ after 1 week of therapy. All through pregnancy, fasting, post-breakfast and post-lunch values were significantly lower with metformin as compared to insulin $(P=<0.001-.036 ; 95 \% \mathrm{Cl}$, 0.53-24.95). However, there was no statistically significant difference in post-dinner values (130 vs. 138; $P=0.316 ; 95 \% \mathrm{Cl}, 3.92-11.92)$

Figure 1 also shows that in the metformin group the glycemic goal ( $<100 \mathrm{mg} / \mathrm{dL}$ for fasting glucose, $<130 \mathrm{mg} / \mathrm{dL}$ for postprandial values) was achieved in all values except post-dinner plasma glucose value. In the insulin group, none of the values reached the glycemic goal.

Considering individual patients, glycemic goal was reached by $24(82 \%)$ and $13(54 \%)$ women on metformin and insulin, respectively. This difference was statistically significant $(P=.024)$. Twelve (40\%) patients on metformin and 18 $(69 \%)$ patients on insulin (Pearson $X^{2}=4.29 ; P=$ 0.038 ) required repeated dose adjustments based on glycemic profile. Metformin thus showed more uniform control of sugars as compared to insulin. Two patients on metformin required additional small dose of insulin at around 36 weeks of gestation.

The incidence of maternal complications such as preeclampsia, UTI, vaginitis, polyhydramnios and preterm labor was comparable between the 2 groups $(P=0.12-1.00)$. There was no difference in the rate and indications for cesarean sections between the 2 groups. Total maternal weight gain in the metformin group $(5.5 \pm 2.9 \mathrm{~kg})$ was significantly less than that in the insulin group $(7.4 \pm 2.8 \mathrm{~kg}, P=0.017)$.

There were no macrosomic babies. Shoulder dystocia was encountered in 1 baby weighing $3.3 \mathrm{~kg}$ in the metformin group. Cephalhematoma noted in the insulin group was after a normal vaginal delivery. Neonatal details [Table 2] show that there were no major differences in neonatal morbidity except for NICU admission. The power of study calculated for NICU stay was $72 \%$. Mean duration of stay for babies in the insulin group was 6.5 (2-20) days; and for babies in the metformin group, it was 2.5 days. Longer NICU stay in the insulin group was because of higher incidence of hyperbilirubinemia / hypoglycemia. Neonatal hypoglycemia significant enough to cause seizures was encountered in 1 baby in the insulin group. No baby had Apgar score $<7$ at birth, and there were no perinatal deaths. Except for 1 major cardiac anomaly requiring surgery in the insulin group for a mother with T2DM, all other anomalies were minor.

\section{Maternal side effects [Table 2]}

There was 1 case of gastritis with metformin, which was corrected with reduction of dose. There were no cases of diabetic ketoacidosis or 
Table 2: Neonatal outcome

\begin{tabular}{lccc}
\hline Group & Metformin $\left(n=31^{*}\right)$ & Insulin $(n=30)$ & $P$ value \\
\hline Mean Gestational Age (weeks) & $38.1 \pm 0.6$ & $37.6 \pm 1.5$ & 0.34 \\
Mean Birth weight (kg) & $2.98 \pm 0.4$ & $3.1 \pm 0.4$ & 0.30 \\
Small for gestational age & $2(6.5 \%)$ & $7(23.3 \%)$ & 0.145 \\
Preterm & $2(6.5 \%)$ & $1(3.3 \%)$ & 0.612 \\
Congenital defects & $2(6.5 \%)$ & $2(6.7 \%)$ & 1.00 \\
NICU stay > 24 hours & $3(9.6 \%)$ & $11(36.7 \%)$ & 0.02 \\
Hypoglycemia & 0 & $2(6.7 \%)$ & 0.49 \\
Hyperbilirubinemia & $9(29 \%)$ & $12(40 \%)$ & 0.39 \\
Polycythemia & 0 & $1(3.3 \%)$ & 1.00 \\
\hline
\end{tabular}

One pair of twins*; $P$ value is computed from Chi-square test, $P=<0.05$

lactic acidosis in this study. Two mothers in the insulin group had symptomatic hypoglycemia

\section{Cost of therapy}

Insulin therapy was considerably more expensive. Cost of therapy per patient per day varied between Rs. 6 and 12 for metformin. It was Rs. 30-145 for insulin, almost 5- to 10-fold even after excluding the cost of syringes and needles.

\section{DISCUSSION}

In this prospective study, we observed that metformin is an effective alternative to insulin in diabetic pregnant women. Insulin improves glycemic profile but does not improve insulin resistance, an important feature of pregnancy, GDM and T2DM. Metformin being an insulin sensitizer targets insulin resistance without enhancing endogenous insulin production, in addition to reducing plasma insulin levels. Glycemic control throughout pregnancy and the number of women attaining glycemic goal were significantly better with metformin as compared to insulin in our population, where daily monitoring of glucose could not be done. Similar results have been noted in other studies. ${ }^{[3]}$ Dose adjustments were required less often with metformin unlike intermediate- acting insulin despite longer duration of action of the latter. Satisfactory glycemic profile was obtained within 1 week with metformin. Coetzee et al. also noted that oral drugs if successful produce maternal normoglycemia within a short time whereas excellent control with insulin may take 2 to 3 weeks of adjustment. ${ }^{[4]}$

Maternal and fetal complications were comparable in both groups except that the SGA babies were more in the insulin group. This has been noted with stringent control of diabetes earlier, but we are unable to explain this as the mean sugar values were comparatively higher in the insulin group. ${ }^{[5]}$

Coetzee et al. tried metformin in 60 diabetic patients, majority of them being maturity onset and only 21 were GDM. ${ }^{[6]}$ The failure rate in the former was $54 \%$ while in the latter it was $29 \%$. This may be because $79 \%$ of their patients were overweight. This study also noted a higher incidence of neonatal hyperbilirubinemia though overall neonatal morbidity was low in the metformin group.

Hellmuth et al. noted a high incidence of preeclampsia and perinatal deaths with metformin. ${ }^{[7]}$ On the contrary, it is now believed 
that metformin may reduce preeclampsia in GDM women by reducing the endothelial activation and maternal inflammatory response of insulin resistance. ${ }^{[3,8]}$ We did not observe increased incidence of preeclampsia with metformin. There were no perinatal deaths in our study, and perinatal morbidity has been similar except for longer NICU stay for babies in the insulin group. Results of MiG trial showed that there was no difference in perinatal outcome in groups treated with metformin or insulin and that women preferred metformin to insulin. So far this has been the largest randomized trial, with 751 women, to assess the efficacy and safety of metformin in women with GDM. ${ }^{[8]}$

Advantages of metformin are its ease of administration and its low cost. This is beneficial in resource-poor populations. Disadvantages of insulin are problems of storage, the inconvenience of daily injections, besides increase in appetite and weight. Dosing of metformin is standardized, unlike insulin, and can be managed by obstetric specialists without the help of general physicians. Metformin does not cause hypoglycemia.

Though metformin crosses placenta, it is not teratogenic. There are no known adverse fetal effects. ${ }^{[8,9]}$ It can be continued for T2DM patients who are being treated preconceptionally.

The limitations of this study are that it is not a randomized controlled trial and the number of participants is small. Management may not have been consistent as the metformin and insulin groups were managed by two different consultants. We have not evaluated the longterm outcomes in infants exposed to metformin.
However, the study by Glueck et al. has shown that neonatal outcomes have been good, with normal growth and development up to 18 months of life..$^{[9]}$ We monitored weekly sugar profile as against the ideal daily self-monitoring of glucose. This was not possible due to the cost involved and reluctance on the part of patients. Nevertheless, there have been no major maternal or perinatal problems. Hence we feel, in resource-poor settings weekly glycemic profile is practical. Glycosylated hemoglobin, which gives an overall view of glucose control, also was not done in all patients.

The convenience and low cost of metformin may improve compliance when applied to a large population. Milder hyperglycemia of GDM in general responds well to metformin. In 2 patients, we had to add a small dose of insulin, late in the third trimester. As insulin requirement is small for this "add-on therapy," the cost is less. A clinical update on metformin therapy in pregnancy in Australia has recommended that metformin use may be considered as an adjunct or alternative to insulin therapy. [10] Though metformin use in pregnancy is not yet popular in India, a preliminary study by Ramachandran et al. reported it is useful either as an adjunct to insulin or even as a monotherapy. ${ }^{[11]}$

\section{CONCLUSION}

We observed that glucose control was better and quicker with metformin than with insulin. Metformin therapy is practical and cheap compared to insulin. Our experience adds to the limited published literature in India on the use of metformin in GDM and T2DM. It 
is a useful first-line therapy that is practical in resource-poor settings.

\section{ACKNOWLEDGMENT}

The authors thank Dr. Pratap K., Head of the Department of OBGYN, for permitting to use his patients on insulin therapy as controls for this study.

\section{REFERENCES}

1. Mohan V, Sandeep S, Deepa R, Shah B, Varghese C. Epidemiology of type 2 diabetes: Indian scenario. Indian J Med Res 2007;125:21730.

2. Carpenter MW, Coustan DR. Criteria for screening tests for gestational diabetes. Am J Obstet Gynecol 1982;144:768-73.

3. Hughes RC, Rowan JA. Pregnancy in women with Type 2 diabetes: who takes metformin and what is the outcome? Diabet Med 2006;23:318-22.

4. Coetzee EJ, Jackson WP. The management of non-insulin - dependent diabetes during pregnancy. Diab Res Clin Pract 1986;1:281-7.

5. Langer O, Levy J, Brustman L, Anyaegbunam A, Merkatz R, Divon M. Glycemic control in gestational diabetes mellitus--how tight is tight enough: small for gestational age versus large for gestational age? Am J Obstet Gynecol 1989;161:646-53.

6. Coetzee EJ, Jackson WP. Metformin in management of pregnant insulin independent Diabetics. Diabetologia 1979;16:241-5.

7. Hellmuth E, Damm P, Mølsted-Pedersen L. Oral hypoglycaemic agents in 118 diabetic pregnancies. Diabet Med 2000;17:507-11.

8. Rowan JA, Hague WM, Gao W, Battin MR, Moore MP. Metformin versus insulin for the treatment of gestational diabetes. N Engl J Med 2008;358:2003-15.

9. Glueck CJ, Goldenberg N, Pranikoff J, Loftspring $M$, Sieve L, Wang P. Height, weight, and motorsocial development during the first 18 months of life in infants born to 109 mothers with polycystic ovary syndrome who conceived on and continued metformin through pregnancy. Hum Reprod 2004;19:1323-30.

10. Simmons D, Walters BN, Rowan JA, Mclntyre DH. Metformin therapy and diabetes in pregnancy Med J Aust 2004;180:462-4.

11. Vijay V, Snehalatha C, Vijayalaskhmi S, Vijay V. Use of metformin in pregnancies with Diabetes: $A$ case series from India. J Assoc Physicians India 2005;53:157-8.

Source of Support: Nil. Conflict of Interest: None declared. 